\title{
Variants Close to NTRK2 Gene Are Associated With Birth Weight in Female Twins
}

\author{
Sarah J. Metrustry, ${ }^{1}$ Mark H. Edwards, ${ }^{2}$ Sarah E. Medland, ${ }^{4}$ John W. Holloway, ${ }^{3}$ \\ Grant W. Montgomery, ${ }^{5}$ Nicholas G. Martin, ${ }^{6}$ Tim D. Spector, ${ }^{1}$ Cyrus Cooper, ${ }^{2,7,8}$ and Ana M. Valdes ${ }^{1,9}$ \\ ${ }^{1}$ Department of Twin Research and Genetic Epidemiology, King's College London, St Thomas' Hospital, London, UK \\ ${ }^{2}$ MRC Lifecourse Epidemiology Unit, University of Southampton, Southampton General Hospital, Southampton, UK \\ ${ }^{3}$ Department of Human Development and Health, Faculty of Medicine, University of Southampton, Southampton, UK \\ ${ }^{4}$ Department of Quantitative Genetics, QIMR Berghofer Medical Research Institute, Brisbane, Queensland, Australia \\ ${ }^{5}$ Department of Molecular Epidemiology, QIMR Berghofer Medical Research Institute, Brisbane, Queensland, Australia \\ ${ }^{6}$ Department of Genetic Epidemiology, QIMR Berghofer Medical Research Institute, Brisbane, Queensland, Australia \\ ${ }^{7}$ NIHR Musculoskeletal BRU, University of Oxford, Nuffield Orthopaedic Centre, Headington, Oxford, UK \\ ${ }^{8}$ National Institute for Health Research Biomedical Research Centre, University of Southampton and University Hospital \\ Southampton NHS Foundation Trust, Southampton \\ ${ }^{9}$ Department of Academic Rheumatology, University of Nottingham, City Hospital, Nottingham, UK
}

\begin{abstract}
Low weight at birth has previously been shown to be associated with a number of adult diseases such as type 2 diabetes, cardiovascular disease, high blood pressure, and obesity later in life. Genome-wide association studies (GWAS) have been published for singleton-born individuals, but the role of genetic variation in birth weight (BW) in twins has not yet been fully investigated. A GWAS was performed in 4,593 female study participants with BW data available from the TwinsUK cohort. A genome-wide significant signal was found in chromosome 9, close to the NTRK2 gene (OMIM: 600456). QIMR, an Australian twin cohort ( $n=$ $3,003)$, and UK-based singleton-birth individuals from the Hertfordshire cohort $(n=2,997)$ were used as replication for the top two single nucleotide polymorphism (SNPs) underpinning this signal, rs 12340987 and rs7849941. The top SNP, rs12340987, was found to be in the same direction in the Australian twins and in the singleton-born females (fixed effects meta-analysis beta $=-0.13, \mathrm{SE}=0.02$, and $p=1.48 \times$ $10^{-8}$ ) but not in the singleton-born males tested. These findings provide an important insight into the genetic component of BW in twins who are normally excluded due to their lower BW when compared with singleton births, as well as the difference in BW between twins. The NTRK2 gene identified in this study has previously been associated with obesity.
\end{abstract}

Keywords: birth weight, twins, GWAS

Birth weight (BW) is an important indicator of overall health and is influenced by a number of factors, including smoking, drinking alcohol during pregnancy, low parental socio-economic status, education levels, low income, and inadequate living conditions (Joloza, 2012). In addition, a wealth of evidence linking low BW to a higher risk of perinatal morbidity and mortality already exists (Battaglia \& Lubchenco, 1967; McIntire et al., 1999), and this has been further extended to show that a low BW can impact on adulthood diseases such as type 2 diabetes, cardiovascular diseases, high blood pressure, and obesity (Barker, 2004; Jarvelin et al., 2004; McIntire et al., 1999). To date, little is actually known about the genetic architecture that determines BW variability, although some recent studies are beginning to shed some light. Six European cohorts $(n=10,263)$ have been studied and the results show that variants in ACDY5 and near to CCNL1 (OMIM: 613384) are associated with fetal growth and BW, and this has been further replicated in an additional 13 studies (Freathy et al., 2010). In addition, maternal inheritance of a repetitive sequence variant of the PHLDA2 gene (OMIM: 602131) has been shown to significantly increase BW (Ishida et al., 2012). The most recent meta-analysis has extended the number of loci associated with BW, at genome-wide significance to seven, accounting for a similar proportion of variance as maternal smoking. Five of the loci are known to be associated with other

RECEIVED 27 April 2014; ACCEPTED 8 May 2014. First published online 20 June 2014.

ADDRESS FOR CORRESPONDENCE: Sarah Metrustry, Department of Twin Research and Genetic Epidemiology, St. Thomas' Hospital, Westminster Bridge Road, London SE1 7EH, UK. E-mail. sarah.metrustry@kcl.ac.uk 
phenotypes: $A D C Y 5$ and $C D K A L 1$ with type 2 diabetes, $A D R B 1$ with adult blood pressure, and HMGA2 and LCORL with adult height. These findings again highlight genetic links between fetal growth and postnatal growth and metabolism (Horikoshi et al., 2013).

All these studies have specifically excluded multiple births due to issues of relatedness or potential nongeneralizability. Only a few have been conducted to date, and findings suggest that LEP and its receptor may act in fetal growth to promote growth, and in twins it is associated with metabolic disease such as development of type 2 diabetes (Souren et al., 2008). Another study has shown that alteration of HERVWE1 methylation affects discordant monozygotic (MZ), dichorionic twin growth (high expression is correlated with small foetus size; Gao et al., 2012). Twins are an essential group to study because multiple births have been on the rise, with an increase of almost 50\% from 1984-2004 in the United Kingdom (98.5\% of these are twins), and they have high rates of complications (www.twinsuk.co.uk). The key assumption is that weight differences in twin pairs come from random differences in access to nutrition; for example, position in uterus/umbilical cord. This is backed up by Cleary-Goldman and D'Alton (2008), who found that singletons and twins develop at the same rate until week 30, at which point the restrictions in uterus size kick in. Dizygotic twins (DZs) only share $\sim 50 \%$ of their genes (like siblings) and they almost always have a separate placenta. It is believed that access to nutrients in DZs may differ due to mass/function of placenta or placental lesions but these effects are usually moderate. On the other hand, $M Z$ twins are formed from the division of a single ovum, post-fertilization and can have one or two chorions. Severe differences in nutritional intake due to positioning and/or umbilical cord insertion can lead to greater weight discrepancies, and therefore a lighter $\mathrm{MZ}$ twin is likely to be genuinely growth-restricted (Torche \& Echevarria, 2011).

Maternal behavior such as smoking status and pregnancy risk factors may influence the weight at birth of a child and thus the twin model accounts for confounders related to mother and pregnancy because twins share the same uterine environment and have the same gestational age. Therefore, the observed effect of BW in twins comes from intra-uterine growth alone.

In this study, we have carried out a genome-wide association (GWA) analysis in 4,593 female study participants with BW data available from the TwinsUK cohort testing the top hit in an independent Australian twin cohort. We have also included replication in a UK-based cohort of singletons in order to investigate whether the effects that we see in twins can also be seen, and if so, to what extent in single births. Since our discovery cohort was British, the Hertfordshire cohort was useful in that there should be no effects of population stratification.

\section{Materials and Methods}

\section{Study Populations}

TwinsUK is the largest registry of adult twins in the United Kingdom. Started in 1992, it currently encompasses approximately 12,000 volunteer twins from all over the United Kingdom (Moayyeri et al., 2013). The data used in this study were collected from female twins who were recruited through a national media campaign as part of the St Thomas' UK adult twin register, initially a cohort of female volunteers. Twins were invited to attend a central clinic where they underwent a physical examination and numerous investigations, including blood pressure measurement. Information on medical history, drug use, lifestyle, and demographic variables was obtained by a standardized nurse-administered questionnaire. Questions on recalled BW (in kilograms or heavier or lighter status) and birth order were supplied two weeks before the interview, and interviewees were encouraged to consult their mothers for answers. Zygosity was assessed by standardized questionnaire, and DNA fingerprinting was used for confirmation (Poulter et al., 1999). In total, 4,593 female participants were studied with regard to BW (converted to kilograms and then $z$-score calculated). The study was approved by St. Thomas' Hospital Research Ethics Committee, and all twins provided informed written consent.

The Hertfordshire Cohort Study (HCS). From 1911 to 1948 in Hertfordshire (UK), each birth was notified by the attending midwife and the BW was recorded. Subsequently, health visitors who saw each child during infancy recorded his or her weight at the age of 1 year. In Hertfordshire, 7,106 individuals born between 1931 and 1939 who were singleton births and had both birth and infant weights recorded, and were still resident in East Hertfordshire in the late 1990s, were traced using the National Health Service Central Registry data and confirmed to be registered with a Hertfordshire general practitioner (GP) by the Hertfordshire Family Health Services Authority (FHSA). Permission was obtained from GPs to contact 6,099 of these individuals; $3,225(53 \%)$ agreed to a home interview with a trained research nurse who collected information on the medical and social history. Subsequently, 2,997 (1,579 men and 1,418 women) attended a clinic for detailed physiological investigations (Phillips et al., 2005; Robinson et al., 2006). The East and North Hertfordshire Ethical Committees granted ethical approval for the study and all participants gave written informed consent.

QIMR. The QIMR participants in the current study were originally recruited in a series of studies examining health and lifestyle factors (Hansell et al., 2008) and in the context of an ongoing study of melanoma risk factors (Zhu et al., 1999, 2007). BW data were collected from twins' parents and via self-report; where available, the maternal report was used in these analyses. Where available, clinical 
measurements of body mass index (BMI; measured in adulthood and calculated as weight divided by height squared $\left.\left[\mathrm{kg} / \mathrm{m}^{2}\right]\right)$ were used, and these data were augmented with the self-report data for those who had not participated in a clinical study. Participants were genotyped in the context of a larger GWA project which resulted in the genotyping of 16,140 individuals (see Medland et al., 2009) using the Illumina 317, 370, and 610 single nucleotide polymorphism (SNP) chips that included data from twins, their siblings, and their parents. Genotype data were screened for genotyping quality (GenCall $<0.7$ ), SNP and individual call rates $(<0.95)$, HWE failure $\left(p<10^{-6}\right)$, and MAF $(<0.01)$. Association analyses were run using Merlin, using the fastassoc option, which corrects for relatedness.

\section{Methods}

TwinsUK Genotyping. The TwinsUK samples were genotyped using a combination of Illumina arrays: HumanHap300 (Richards et al., 2008; Soranzo et al., 2009), HumanHap610Q, 1M-Duo and 1.2M-Duo 1M. The normalized intensity data for each of the three arrays were pooled separately (with $1 \mathrm{M}$-Duo and $1.2 \mathrm{M}$-Duo $1 \mathrm{M}$ pooled together). For each dataset we used the Illluminus calling algorithm (Teo et al., 2007) to assign genotypes in the pooled data. No calls were assigned if an individual's most likely genotype was called with less than a posterior probability threshold of 0.95 . Validation of pooling was achieved via a visual inspection of 100 random, shared SNPs for overt batch effects. Finally, intensity cluster plots of significant SNPs were visually inspected for over-dispersion biased no calling, and/or erroneous genotype assignment. SNPs exhibiting any of these characteristics were discarded. We applied similar exclusion criteria to each of the three dataset separately. For the samples: Exclusion criteria were (1) sample call rate $<98 \%$, (2) heterozygosity across all SNPs $\geq 2$ standard deviation $(S D)$ from the sample mean $(M),(3)$ evidence of non-European ancestry as assessed by the Principal Component Analysis (PCA) comparison with HapMap3 populations, (4) observed pair-wise identity-by-descent (IBD) probabilities suggestive of sample identity errors; and (5) we corrected misclassified MZ and DZ twins based on IBD probabilities. SNPs: Exclusion criteria were (1) HardyWeinberg $p$-value $<10^{-6}$, assessed in a set of unrelated samples; (2) MAF $<1 \%$, assessed in a set of unrelated samples; and (3) SNP call rate $<97 \%$ (SNPs with MAF $\geq 5 \%$ ) or $<99 \%$ (for $1 \% \leq \mathrm{MAF}<5 \%$ ). Alleles of all three datasets were aligned to HapMap2 or HapMap3 forward strand alleles. Prior to merging, we performed pair-wise comparison among the three datasets and further excluded SNPs and samples to avoid spurious genotyping effects, indentified as follows: (1) concordance at duplicate samples $<1 \%$; (2) concordance at duplicate SNPs $<1 \%$; (3) visual inspection of Quantile-Quantile (QQ) plots for logistic regression applied to all pair-wise dataset comparisons; (4) Hardy-Weinberg $p$-value $<10^{-6}$, assessed in a set of un- related samples; and (5) observed pair-wise IBD probabilities suggestive of sample identity errors. We then merged the three datasets, keeping individuals typed at the largest number of SNPs when an individual was typed at two different arrays. The merged dataset consists of 5,654 individuals (2,040 from the HumanHap300, 3,461 from the HumanHap610Q, and 153 from the HumanHap1M and 1.M arrays) and up to 874,733 SNPs depending on the dataset (HumanHap300: 303,940, HumanHap610Q: 553,487, and HumanHap1M and 1.M: 874,733).

Imputation. This was performed using the IMPUTE software package (v2; Howie et al., 2009) using two reference panels, P0 (HapMap2, rel 22, combined CEU+YRI+ASN panels) and $\mathrm{P} 1(610 \mathrm{k}+$, including the combined HumanHap610k and $1 \mathrm{M}$ reduced to $610 \mathrm{k}$ SNP content).

Replication genotyping. Genotyping in the samples from HCS was carried out by Kbioscience Ltd, Hertfordshire UK. SNPs were genotyped using the KASPar chemistry, which is a competitive allele-specific polymerase chain reaction (PCR) SNP genotyping system using fluorescence resonance energy transfer (FRET) quencher cassette oligos (http://www.lgcgenomics.com/).

Phenotype preparation. We ran a preliminary association on raw BW values and then BW was transformed to a $z$-score ((BW value - mean BW)/SD BW) to facilitate comparison of twins with HCS (results in this manuscript are the $z$-score values). BMI was calculated by weight divided by height squared $\left(\mathrm{kg} / \mathrm{m}^{2}\right)$.

Analyses. The GWA analysis was performed on imputed data using GenABEL (Aulchenko et al., 2007) for twins (to account for relatedness) and PLINK (Purcell et al., 2007) for the HCS samples. We analyzed all twins together, separated by sex and, in addition, separated by zygotic status. Summary statistics (the regression coefficient beta and its standard error) for each cohort were then meta-analyzed using fixed effect models. The 'meta' package for $\mathrm{R}$ was used (R Development Core Team, 2010).

\section{Results}

The descriptive characteristics of the discovery and replication cohorts are shown in Table 1 . The average difference in weight at birth between twins is $0.54 \mathrm{~kg}$ (MZ average is $0.54 \mathrm{~kg}$, while DZ twins were, on average, $0.55 \mathrm{~kg}$ apart at birth).

Given the sample size, the statistical power to identify as genome-wide significant (GWS; $p<5 \times 10^{-8}$ ) in the discovery cohort was $80 \%$ for an effect size of 0.20 given a minor allele frequency (MAF) of 0.13 . The power was $80 \%$ in the discovery plus replication cohorts for an effect size of 0.15 (measured in units of standard deviations from the mean). 


\section{TABLE 1}

Descriptive Statistics of TwinsUK Discovery Cohort and the QIMR Twins Plus Hertfordshire Replication Cohorts

\begin{tabular}{|c|c|c|c|c|c|c|}
\hline Cohort & Description & Sample size & Average BW $(\mathrm{kg})$ & $\mathrm{BW}(S D)$ & Average BMI & BMI (SD) \\
\hline TwinsUK & Females only & 4,593 & 2.42 & 0.58 & 25.31 & 4.68 \\
\hline TwinsUK & $\mathrm{MZ}$ and female & 1,803 & 2.34 & 0.58 & $25.14(n=1,689)$ & $4.81(n=1,689)$ \\
\hline TwinsUK & $\mathrm{DZ}$ and female & 2,783 & 2.47 & 0.58 & $25.23(n=2,748)$ & $4.64(n=2,748)$ \\
\hline QIMR & Females only & 3,033 & 2.49 & 0.57 & 24.77 & 4.79 \\
\hline QIMR & $\mathrm{MZ}$ and female & 1,519 & 2.42 & 0.56 & 24.62 & 4.64 \\
\hline QIMR & DZ and female & 1,514 & 2.57 & 0.56 & 24.93 & 4.92 \\
\hline $\mathrm{HCS}$ & All & 2,997 & 3.43 & 0.53 & 27.37 & 4.35 \\
\hline HCS & Female & 1,418 & 3.35 & 0.50 & 27.61 & 4.91 \\
\hline HCS & Male & 1,579 & 3.50 & 0.54 & 27.16 & 3.77 \\
\hline
\end{tabular}

We ran a preliminary association using raw BW data and found four GWS SNPs at a locus on chromosome 9 compared with the null expectation of no association (Supplementary Table 4). In order to compare with other data available, we then converted the weight at birth to a $z$-score and reran the association. This GWA analysis on the TwinsUK cohort maintained a GWS signal $\left(p<5 \times 10^{-8}\right)$. Creation of residuals to adjust for BMI and also running BMI as a covariate did not alter these results (Supplementary Table 5 and Figure 1).

Our signal achieving GWS in twins lies on chromosome 9, close to NTRK2 (neurotrophic tyrosine kinase, receptor, type 2), which is a membrane-bound receptor, OMIM: 600456 (Figure 2). This gene encodes a member of the neurotrophic tyrosine receptor kinase (NTRK) family. This kinase, upon neurotrophin binding, phosphorylates itself and members of the MAPK pathway. Signaling through this kinase leads to cell differentiation, and mutations in this gene have been associated with obesity and mood disorders (Gray et al., 2007; Yeo et al., 2004). We further examined whether this result was seen predominantly in identical or non-identical twins (Supplementary Table 3) or was due to the twin with the highest or the lowest BW in a pair (Supplementary Table 2). We found that the SNPs were more highly associated with the low BW twin and the effect was larger in $\mathrm{MZ}$ twins.

As NTRK2 has been shown to be related to obesity, we also explored whether these variants were associated with other phenotypes related to adult weight (Supplementary Table 1). We investigated the effect of adult fat mass and percentage body fat on our top signals. Within the TwinsUK sample we found our two top variants were marginally associated with fat mass (rs7849941, $p=.002$; rs12340987, $p=.04)$ and percentage body fat (rs7849941, $p=.0004$; rs12340987, $p=.05)$.

\section{Replication}

We then investigated whether these variants were associated with BW in an independent cohort of UK-based singletons in order to investigate how twins would compare with singleton births with regard to BW genetics. The two top signals, rs7849941 and rs12340987, $\left(r^{2}=0.85\right.$ in 1000 Genomes Project) were selected for replication in HCS, which included both genders; rs7849941 was found to be significantly associated with BW among HCS females ( $p$ $<.04)$ and rs12340987 achieved $p<.08$ among females (Table 2). On the other hand, the effect size among males was in the opposite direction and was not statistically significant for either SNP. A fixed effect meta-analysis of females in TwinsUK and HCS was conducted, and while rs 12340987 achieves suggestive significance, rs7849941, with which it is in strong linkage disequilibrium (LD), shows nominal significance; further, it achieves GWS when the discovery and replication cohorts are combined. As such, there is cumulative evidence that this locus is indeed associated with BW.

The two top SNPs were genotyped for replication in the Hertfordshire cohort $(n=2,997)$ and also followed up in the Australian twin cohort, where they were on a chip $(n=3,003)$. Table 2 shows the genetic association effect size estimates in the discovery and replication cohorts and summary statistic from fixed effect meta-analysis. Effect sizes are standardized and are per standard deviation from the mean. No significant inter-study heterogeneity was observed $(\mathrm{Q}=$ $2.38, p<.123)$ and the between-study variance was $\tau^{2}=$ 0.0023 .

Finally, we compared our results with the published data from the Early Growth Genetics (EGG) consortium (GWA studies (GWAS) on BW in 26,863 singleton-birth individuals of both genders; Horikoshi et al., 2013). SNP rs7849941 is not available in the EGG consortium. Allele A for SNP rs12340987 is not significantly associated with BW and has an effect size of 0.02 (95\% CI -0.001, 0.043), which is consistent with what we see in the HCS study when we combine males and females. Unfortunately, gender-stratified analyses are not publically available.

\section{Discussion}

In this study, we have investigated the role of genetic variation in weight at birth in multiple births. We find that variants mapping to the NTRK2 gene are associated with BW in twins and this effect is seen in both identical and non-identical twins. When the analysis was repeated using only the twin with the highest BW or only the twin with the lowest weight at birth, the variants maintain association, particularly in the low BW twin. 
(a)

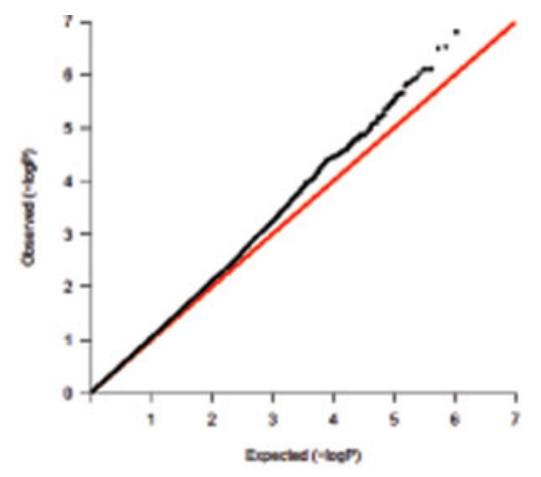

(b)

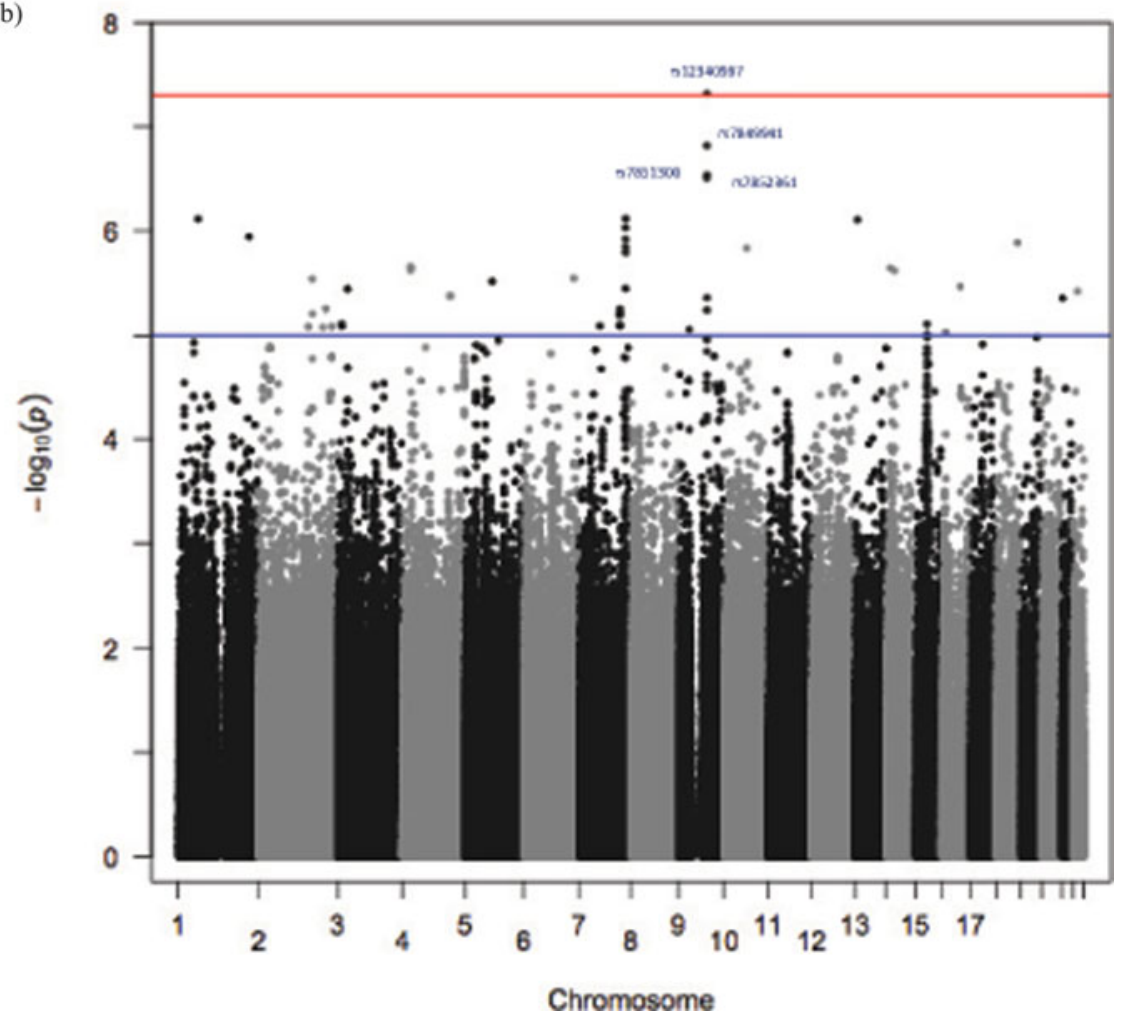

FIGURE 1

(Colour online) TwinsUK: (a) QQ plot; (b) Manhattan plot.

The findings of this study provide a novel insight into the genetic component of BW. The variants discovered to be associated with twin BW are close to the NTRK2 gene that has previously been associated with severe early onset obesity (Gray et al., 2007). Mutations in NTRK2 have been found to be a cause of obesity hyperphagia and developmental delay (OHPDD; Yeo et al., 2004). OHPDD is a disorder characterized by early-onset obesity, hyperphagia, and severe developmental delay in motor function, speech, and language. The association with BW reported by us supports a developmental role for this gene. In addition, variation in the NTRK2 gene has also been implicated in anorexia nervosa and minimum BMI (Ribases et al., 2005), linking this gene also to adult disease. However, the variant identified in our study is not in LD with the variants associated with OHPDD, nor with those associated with anorexia nervosa (maximum $r^{2}=0.04$, data not shown). The other gene near the signal identified is $A G T P B P 1$, a zinc carboxypeptidase that contains nuclear localization signals and an ATP/GTPbinding motif. According to HapMap (www.hapmap.org), there are no GWAS hits mapping to this gene.

Our results show that SNPs close to NTRK2 are significant in females but not in males, with one reason being that boys weigh $136 \mathrm{~g}$ more than girls at term birth (Lehre et al., 2013). The reason for this remains unknown, with many different theories. Proposed modes of action include the action of androgens (de Zegher et al., 1998), the sexual bias in metabolism (Crawford et al., 1987; Lovejoy et al., 
TABLE 2

Genetic Association Effect Size Estimates in the Discovery and Replication Cohorts and Summary Statistic From Fixed Effect Meta-Analysis

\begin{tabular}{|c|c|c|c|c|c|c|c|c|}
\hline SNP & Effect allele & Non-effect allele & Cohort & Gender & MAF & Beta & SE & $p$-value \\
\hline \multirow[t]{6}{*}{ rs7849941 } & \multirow[t]{4}{*}{ A } & \multirow[t]{4}{*}{$\mathrm{T}$} & TwinsUK & $\mathrm{F}$ & 0.13 & -0.17 & 0.03 & 1.53E-07 \\
\hline & & & $\mathrm{HCS}$ & $\mathrm{F}$ & 0.13 & -0.11 & 0.05 & 0.04 \\
\hline & & & $\mathrm{HCS}$ & M & 0.13 & 0.08 & 0.06 & 0.13 \\
\hline & & & QIMR & $\mathrm{F}$ & 0.13 & -0.03 & 0.04 & 0.41 \\
\hline & \multicolumn{3}{|c|}{ TwinsUK+HCS meta-analysis } & $\mathrm{F}$ & 0.13 & -0.16 & 0.03 & 2.83E-08 \\
\hline & \multicolumn{3}{|c|}{ TwinsUK+QIMR+HCS meta-analysis } & $\mathrm{F}$ & 0.13 & -0.12 & 0.02 & $5.46 \mathrm{E}-08$ \\
\hline \multirow[t]{6}{*}{ rs12340987 } & \multirow[t]{4}{*}{ A } & \multirow[t]{4}{*}{ G } & TwinsUK & $\mathrm{F}$ & 0.20 & -0.18 & 0.03 & $4.81 \mathrm{E}-08$ \\
\hline & & & $\mathrm{HCS}$ & $\mathrm{F}$ & 0.15 & -0.09 & 0.05 & 0.08 \\
\hline & & & $\mathrm{HCS}$ & M & 0.15 & 0.08 & 0.05 & 0.14 \\
\hline & & & QIMR & $\mathrm{F}$ & 0.15 & -0.04 & 0.04 & 0.30 \\
\hline & \multicolumn{3}{|c|}{ TwinsUK+HCS meta-analysis } & $\mathrm{F}$ & & -0.16 & 0.03 & $2.72 \mathrm{E}-08$ \\
\hline & \multicolumn{3}{|c|}{ TwinsUK+QIMR+HCS meta-analysis } & $\mathrm{F}$ & & -0.13 & 0.02 & $1.48 \mathrm{E}-08$ \\
\hline
\end{tabular}

Note: Effect sizes are standardized and are per standard deviation from the mean. The two top SNPs were genotyped for replication in the Hertfordshire cohort $(n=2,997)$ and replicated in the Australian Twin (QIMR) cohort $(n=3,033)$.

\section{TwinsUK Regional Plot}

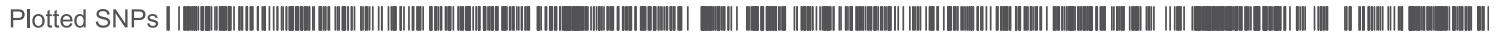

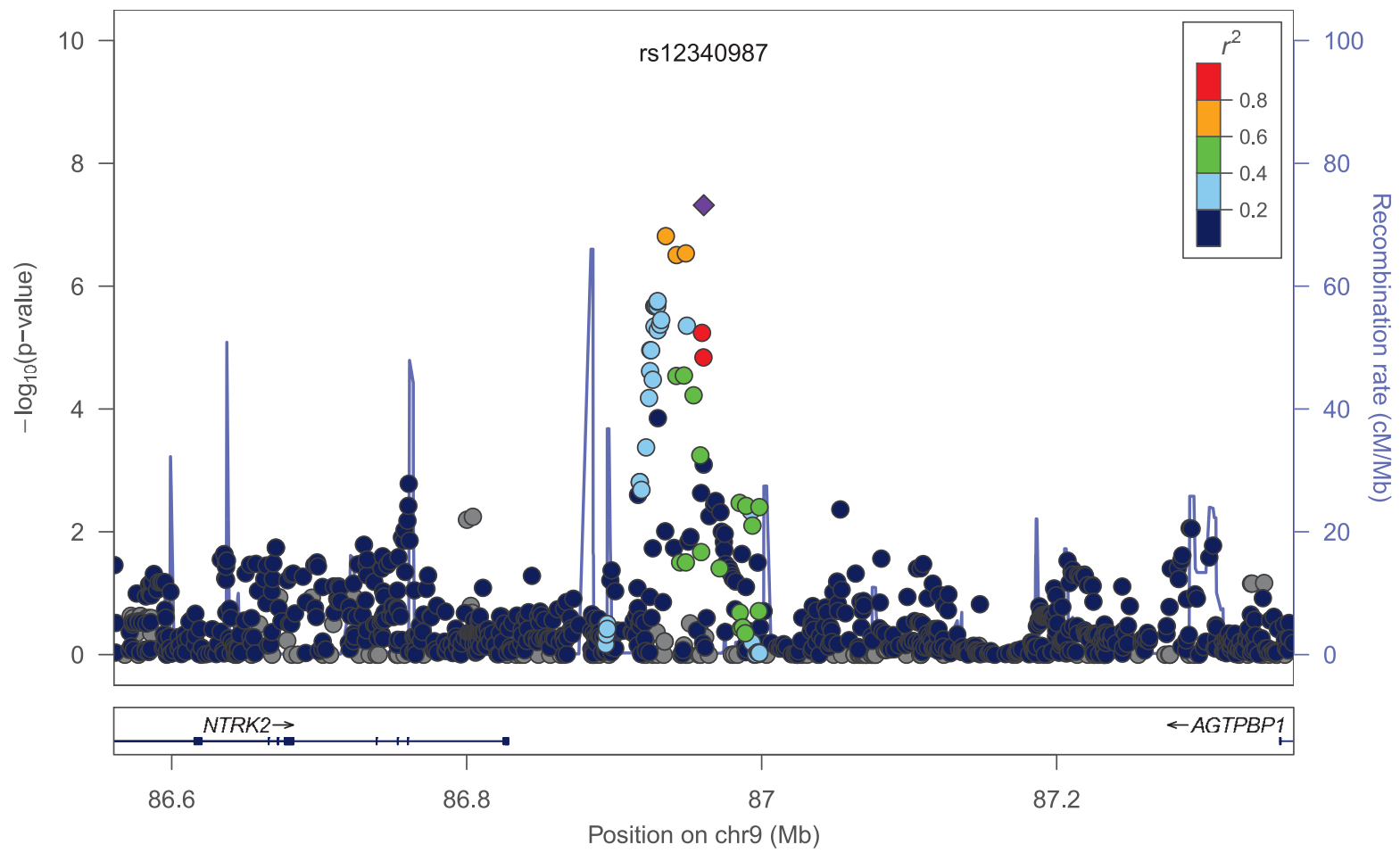

\section{FIGURE 2}

(Colour online) TwinsUK regional plot.

2009), or that boys weigh more due to an undetermined cause that is Y-chromosome-related (Ounsted \& Ounsted, 1970).

Our study was limited by the number of twins available for study, and also because TwinsUK predominantly comprises women, our discovery cohort was limited to women. As BW is only one of many developmental phenotypes, our findings may suggest that a different genetic architecture may be at play in determining female BW than in males.

\section{Supplementary Material}

To view supplementary material for this article, please visit http://dx.doi.org/10.1017/thg.2014.34.

\section{Acknowledgments}

The TwinsUK study was funded by the Wellcome Trust; European Community's Seventh Framework Programme (FP7/2007-2013). The study also receives support from 
the National Institute for Health Research (NIHR)-funded BioResource, Clinical Research Facility, and Biomedical Research Centre based at Guy's and St Thomas' NHS Foundation Trust in partnership with King's College London. SNP Genotyping was performed by the Wellcome Trust Sanger Institute and the National Eye Institute via NIH/CIDR. The research leading to these results has received funding from the European Community's Seventh Framework Programme under grant No. 277849 (www.eurhealth.org). The Hertfordshire Cohort Study was funded by the Medical Research Council of Great Britain; Arthritis Research UK; the International Osteoporosis Foundation; as well as the NIHR Musculoskeletal Biomedical Research Unit, University of Oxford and the NIHR Nutrition Biomedical Research Centre, University of Southampton. QIMR: We thank the twins and their families for their participation, Dixie Statham, Ann Eldridge, Marlene Grace, and Kerrie McAloney (sample collection); Lisa Bowdler, Steven Crooks (DNA processing), David Smyth, Harry Beeby, and Daniel Park (IT support). Funding: Australian National Health and Medical Research Council (241944, 339462, 389927, 389875, 389891, 389892, 389938, 442915, 442981, 496739, 552485, 552498); Australian Research Council (A7960034, A79906588, A79801419, DP0770096, DP0212016, DP0343921); FP-5 GenomEUtwin Project (QLG2-CT-2002-01254); US National Institutes of Health (NIH grants AA07535, AA10248, AA13320, AA13321, AA13326, AA14041, MH66206). SEM is supported by an ARC Future Fellowship. Role of the funding source: The European Commission had no role in the study design, data collection, data analysis, data interpretation, or the writing of the report.

\section{Abbreviations}

ASN: East Asian population

CEU: Utah residents with North European ancestry

KASP: Kompetitive Allele Specific PCR

OMIM: Online Mendelian Inheritance in Man

YRI: Yoruba from Nigeria.

\section{References}

Aulchenko, Y. S., Ripke, S., Isaacs, A., \& van Duijn, C. M. (2007). GenABEL: An R library for genome-wide association analysis. Bioinformatics, 23, 1294-1296.

Barker, D. J. (2004). The developmental origins of adult disease. Journal of the American College of Nutrition, 23, 588S595S.

Battaglia, F. C., \& Lubchenco, L. O. (1967). A practical classification of newborn infants by weight and gestational age. The Journal of Pediatrics, 71, 159-163.

Cleary-Goldman, J., \& D'Alton, M. E. (2008). Growth abnormalities and multiple gestations. Seminars in Perinatology, 32, 206-212.
Crawford, M. A., Doyle, W., \& Meadows, N. (1987). Gender differences at birth and differences in fetal growth. Human Reproduction, 2(6), 517-520.

de Zegher, F., Francois, I., Boehmer, A. L., Saggese, G., Muller, J., Hiort, O., . . S Sippell, W. G. (1998). Androgens and fetal growth. Hormone Research, 50(4), 243-244.

Freathy, R. M., Mook-Kanamori, D. O., Sovio, U., Prokopenko, I., Timpson, N. J., Berry, D. J., . . McCarthy, M. I. (2010). Variants in ADCY5 and near CCNL1 are associated with fetal growth and birth weight. Nature Genetics, 42, 430435.

Gao, Y., He, Z., Wang, Z., Luo, Y., Sun, H., Zhou, Y., ... Jiang, S. (2012). Increased expression and altered methylation of HERVWE1 in the human placentas of smaller fetuses from monozygotic, dichorionic, discordant twins. PLoS One, 7, e33503.

Gray, J., Yeo, G., Hung, C., Keogh, J., Clayton, P., Banerjee, K., ... Farooqi, I. S. (2007). Functional characterization of human NTRK2 mutations identified in patients with severe early-onset obesity. International Journal of Obesity (London), 31, 359-364.

Hansell, N. K., Agrawal, A., Whitfield, J. B., Morley, K. I., Zhu, G., Lind, P. A., ... Martin, N. G. (2008). Long-term stability and heritability of telephone interview measures of alcohol consumption and dependence. Twin Research and Human Genetics, 11, 287-305.

Horikoshi, M., Yaghootkar, H., Mook-Kanamori, D. O., Sovio, U., Taal, H. R., Hennig, B. J., ... Early Growth Genetics, Consortium. (2013). New loci associated with birth weight identify genetic links between intrauterine growth and adult height and metabolism. Nature Genetics, 45, 7682.

Howie, B. N., Donnelly, P., \& Marchini, J. (2009). A flexible and accurate genotype imputation method for the next generation of genome-wide association studies. PLoS Genetics, 5, e1000529.

Ishida, M., Monk, D., Duncan, A. J., Abu-Amero, S., Chong, J., Ring, S. M., ... Moore, G. E. (2012). Maternal inheritance of a promoter variant in the imprinted PHLDA2 gene significantly increases birth weight. American Journal of Human Genetics, 90, 715-719.

Jarvelin, M. R., Sovio, U., King, V., Lauren, L., Xu, B., McCarthy, M. I., ... Elliott, P. (2004). Early life factors and blood pressure at age 31 years in the 1966 northern Finland birth cohort. Hypertension, 44, 838-846.

Joloza, T. (2012). Measuring National Wellbeing - Children's Wellbeing. Newport, South Wales: Office for National Statistics.

Lehre, A. C., Laake, P., \& Sexton, J. A. (2013). Differences in birth weight by sex using adjusted quantile distance functions. Statistics in Medicine, 32, 2962-2970.

Lovejoy, J. C., Sainsbury, A., \& Stock Conference Working, Group. (2009). Sex differences in obesity and the regulation of energy homeostasis. Obesity Reviews, 10, 154-167.

McIntire, D. D., Bloom, S. L., Casey, B. M., \& Leveno, K. J. (1999). Birth weight in relation to morbidity and mortality among newborn infants. New England Journal of Medicine, 340(16), 1234-1238. 
Medland, S. E., Nyholt, D. R., Painter, J. N., McEvoy, B. P., McRae, A. F., Zhu, G., .. . Martin, N. G. (2009). Common variants in the trichohyalin gene are associated with straight hair in Europeans. American Journal of Human Genetics, 85, 750-755.

Moayyeri, A., Hammond, C. J., Valdes, A. M., \& Spector, T. D. (2013). Cohort profile: TwinsUK and Healthy Ageing Twin Study. International Journal of Epidemiology, 42, 76-85.

Ounsted, C., \& Ounsted, M. (1970). Effect of Y chromosome on fetal growth-rate. Lancet, 2, 857-858.

Phillips, D. I., Goulden, P., Syddall, H. E., Aihie Sayer, A., Dennison, E. M., Martin, H., ... Hertfordshire Cohort Study Group. (2005). Fetal and infant growth and glucose tolerance in the Hertfordshire Cohort Study: A study of men and women born between 1931 and 1939. Diabetes, 54, S145-150.

Poulter, N. R., Chang, C. L., MacGregor, A. J., Snieder, H., \& Spector, T. D. (1999). Association between birth weight and adult blood pressure in twins: Historical cohort study. British Medical Journal, 319, 1330-1333.

Purcell, S., Neale, B., Todd-Brown, K., Thomas, L., Ferreira, M. A., Bender, D., ... Sham, P. C. (2007). PLINK: A tool set for whole-genome association and population-based linkage analyses. American Journal of Human Genetics, 81, 559-575.

R Development Core Team. (2010). R: A language and environment for statistical computing. Retrieved from http://www.R-project.org/

Ribases, M., Gratacos, M., Badia, A., Jimenez, L., Solano, R., Vallejo, J., ... Estivill, X. (2005). Contribution of NTRK2 to the genetic susceptibility to anorexia nervosa, harm avoidance and minimum body mass index. Molecular Psychiatry, $10,851-860$.

Richards, J. B., Rivadeneira, F., Inouye, M., Pastinen, T. M., Soranzo, N., Wilson, S. G., ... Spector, T. D. (2008). Bone mineral density, osteoporosis, and osteoporotic fractures: A genome-wide association study. Lancet, 371, 1505-1512.

Robinson, S. M., Batelaan, S. F., Syddall, H. E., Sayer, A. A., Dennison, E. M., Martin, H. J., ... Hertfordshire Cohort
Study. (2006). Combined effects of dietary fat and birth weight on serum cholesterol concentrations: The Hertfordshire Cohort Study. American Journal of Clinical Nutrition, 84, 237-244.

Soranzo, N., Rivadeneira, F., Chinappen-Horsley, U., Malkina, I., Richards, J. B., Hammond, N., ... Deloukas, P. (2009). Meta-analysis of genome-wide scans for human adult stature identifies novel Loci and associations with measures of skeletal frame size. PLoS Genetics, 5, e1000445.

Souren, N. Y., Paulussen, A. D., Steyls, A., Loos, R. J., Stassen, A. P., Gielen, M., ... Zeegers, M. P. (2008). Common SNPs in LEP and LEPR associated with birth weight and type 2 diabetes-related metabolic risk factors in twins. International Journal of Obesity (London), 32, 12331239.

Teo, Y. Y., Inouye, M., Small, K. S., Gwilliam, R., Deloukas, P., Kwiatkowski, D. P., \& Clark, T. G. (2007). A genotype calling algorithm for the Illumina BeadArray platform. Bioinformatics, 23, 2741-2746.

Torche, F., \& Echevarria, G. (2011). The effect of birthweight on childhood cognitive development in a middle-income country. International Journal of Epidemiology, 40(4), 10081018.

Yeo, G. S., Connie Hung, C. C., Rochford, J., Keogh, J., Gray, J., Sivaramakrishnan, S., ... Farooqi, I. S. (2004). A de novo mutation affecting human TrkB associated with severe obesity and developmental delay. Nature Neuroscience, 7, 1187-1189.

Zhu, G., Duffy, D. L., Eldridge, A., Grace, M., Mayne, C., O’Gorman, L., ... Martin, N. G. (1999). A major quantitative-trait locus for mole density is linked to the familial melanoma gene CDKN2A: A maximum-likelihood combined linkage and association analysis in twins and their sibs. American Journal of Human Genetics, 65, 483492.

Zhu, G., Montgomery, G. W., James, M. R., Trent, J. M., Hayward, N. K., Martin, N. G., \& Duffy, D. L. (2007). A genome-wide scan for naevus count: Linkage to CDKN2A and to other chromosome regions. European Journal of Human Genetics, 15, 94-102. 